\title{
The Dof-Box Project: An Educational Kit for Configurable Robots
}

\author{
D. Daidié, O. Barbey, A. Guignard, D. Roussy, F. Guenter, A. Ijspeert, A. Billard. \\ LASA Laboratory, School of Engineering, EPFL, Lausanne CH 1015, Switzerland \\ Contact: david.dadie@epfl.ch, aude.billard@epfl.ch
}

\begin{abstract}
This paper describes the Dof-Box, a modular, reconfigurable robot, used in Hands-on practicals of robotics taught to microengineering students. We present the hardware and software of the robots and describe the pedagogical approach adopted in the practicals. This approach encourages the creativity and imagination of the students, while ensuring that the students put in practice fundamental laws of control they are taught in class. Some of the results, including particular design of robots, obtained by the students are also shown.
\end{abstract}

Index Terms - education robotics, modular robots, reconfigurable robots.

\section{INTRODUCTION}

$\mathrm{T}^{\mathrm{s}}$ He Dof-Box (Degrees of Freedom Box) is an educational robotic kit intended to allow engineering students to get a first hands-on experience of robotics. The Dof-Box kit is composed of a set of five to seven one-degree-of-freedom modules. Students may assemble each of the modules as they wish so as to create an arbitrary shape, which they may then control; the sole criteria for the design being that the robot should move forward on a flat surface. Through the design, building and eventually controlling the robot, students get to put in practice concepts of mechanics, regulation and dynamical system control they have studied theoretically in class. In addition to enabling the students to experiment with the control of robots, this project encourages them to use their creativity and imagination in the design of the robot's body.

Each Dof-Box module has a single degree of freedom (dof) and is endowed with a control board. It has eight mechanical connections, allowing it to be mechanically connected to other similar modules. This modularity allows for a very wide variety of robot shapes. The various modules can communicate with one-another and with the main $\mathrm{PC}$ through the $\mathrm{I} 2 \mathrm{C}$ or $\mathrm{CAN}$ protocols. A power bus runs through all the modules, providing them with the necessary voltage. The power control and communication electronics are all integrated on mechanical structure of the Dof-Box. It also has a DC motor and a reduction gearbox, which enables it to produce a torque of over $1[\mathrm{Nm}]$.

For the students, the aim is to use five to seven Dof-Boxes modules and build and control a robot able to move a flat surface.

\section{RELATED WORK}

A large number of modular or reconfigurable robot kits are used in universities or are available on the market. For example the "M-TRAN" [4] is a self-reconfigurable modular robot. It consists of three mechanical parts serially linked through two motorized rotative joints. It is autonomous in the sense that it has on-board batteries and supports wireless communication.

The "PolyBot" [3] is another modular self-reconfigurable robot. Each module has a single dof and can generate high torques with a high precision (less and 0.04 degrees error).

The « Coro » [5] module is also a modular self-reconfigurable robot. It has two dofs and a higher number of mechanical connections, enabling it to have one-to-many connections. This allows the robot to easily switch across configurations without losing modules.

There are also many robots intended for educational or purposes. Most of them are wheeled robots such as the « epuck »[6], which is a new two-wheel robot developed at EPFL. It has many sensors such as a VGA camera, IR distance sensors, and an accelerometer. It uses a C-programmable DsPic controller and supports wireless communication through Bluetooth.

All the « e-puck» project is open source and open hardware. One can find all the details about the realisation and software on [6].The Dof-Box project will also be available on a web site soon.

Although modular robots or educational robots are widely used, the Dof-Box combines those two characteristics. Like the above educational robot kit, and in contrast to other modular robot kits, the Dof-Box has been designed so as to provide an educational tool. It is thus cheaper and, hence, less precise than other reconfigurable and modular robots, but provides much more flexibility in the control system than those. 


\section{DOF-BOX HARDWARE AND SOFTWARE}

A. Mechanical components

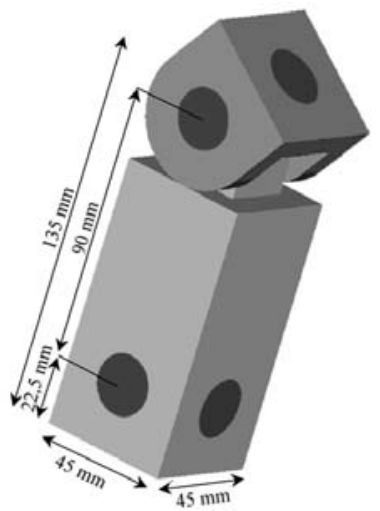

Figure 1: Dof-Box simple DAO shape. The circles indicate the position of mechanical connections.

The general shape of the Dof-Box module is shown in Figure 1. It consists of a parallelepiped with a square basis, whose top can rotate around an axis parallel to an edge of the basis. The dimensions of the parallelepiped is $a \times a \times 3 a$ so that the rotating top forms a cube.

Each face is endowed with mechanical connections (shown in red in Figure 1) allowing multiple Dof-Boxes to be connected to each other. Those connections are located at the center of the bottom and top square basis and at the center of the lower third of each rectangular face of the parallelepiped. The rotating top has similar connections centred on its rotation axis. This enables the Dof-Boxes to be assembled in closed forms and keep the global parallelepiped shape when the top is undergoes a 90 degrees rotation.
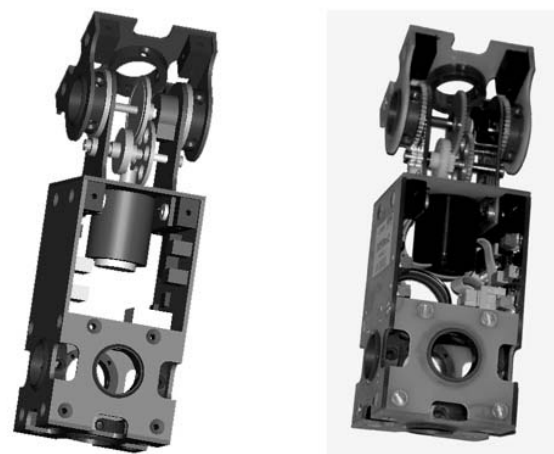

Figure 2: Dof-Box detailed DAO and real shape

Efforts were made to have as much open space as possible, to enable access to electrical connectors and to simplify the routing of cables between connected Dof-Box modules. This is important to avoid mechanical interference between the cables and the rotating parts.
The complete Dof-Box module has a size $45 \times 45 \times 135[\mathrm{~mm}]$. It weighs 180 [g] and its top has a rotating range of [-90 90] degrees. It can reach an angular velocity of about $4[\mathrm{rad} / \mathrm{s}]$.

\section{1) Mechanical connections}

As described above, on each face of the Dof-Box, there is at least one mechanical connector. Those connectors are meant to connect two Dof-Box modules together. They are all of the "female" type and consist of PVC cylinders with four holes evenly distributed on their middle cross-section, as shown in Figure 3.

To connect two modules together one uses an additional connector of the "male type" which connects to the two modules. This connector is an aluminium tube, which can be inserted into the female connectors. It has eight radially distributed bore holes at each end. The two connectors can thus be screwed together as shown in Figure 4. This way any connector of a module can be connected to any connector of another module in eight different orientations.

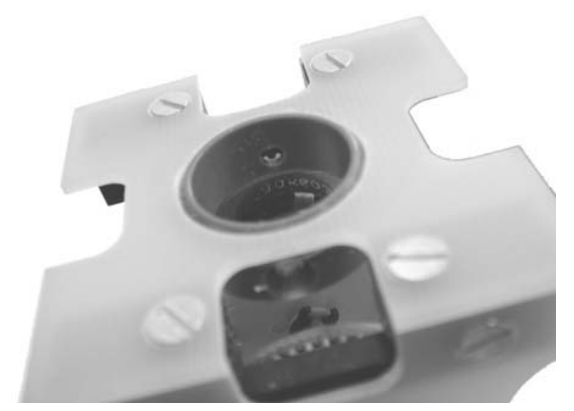

Figure 3: Mechanical connection, of "female” type.

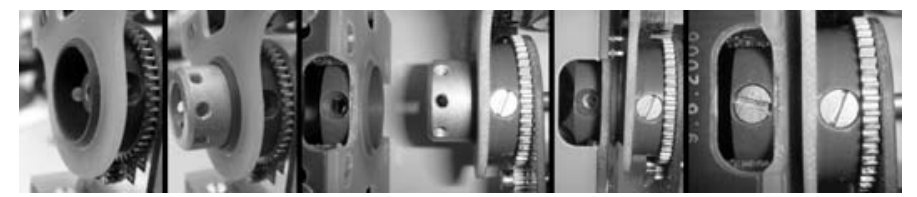

Figure 4: Connecting two modules.

2) Hardware integration

All the carrying structure of the Dof-Box is made of PCBs. This reduces the size and weight of the module, while remaining easy to machine and assemble. The two largest faces carry all the electronics. One of the PCB carrying the reduction gearbox also carries the potentiometer. The other PCBs bear no electronics.

\section{3) Motorization}

A Faulhaber 2224012SR DC motor is used with a 12[V] or $15[\mathrm{~V}] \mathrm{DC}$ power supply. It has the following characteristics :

- Nominal voltage: $\quad \mathrm{U} 0=12[\mathrm{~V}]$

- Resistance : $\quad \operatorname{Rmot}=8,71[\Omega]$

- Troque constant : $\quad \mathrm{k}_{\mathrm{M}}=14,5[\mathrm{mNm} / \mathrm{A}]$ 


\section{4) Reduction gear box}

The reduction is achieved through four successive reduction stages distributed along two parallel axes (except the motor one). A picture of the reduction gearbox is displayed in Figure 5 . The numbers in parentheses in the next paragraph refer to the numbers on Figure 5.

The first stage of the reduction gear is made of the 10-teeth cogwheel and a 30 -teeth gear wheel (1). This stage brings the rotation axis in the right direction. The following stages are composed of three other cogwheel-gear wheel pairs, each of them having a reduction ratio of four. The total reduction ratio is thus 192. The last stage is a parallel one; two cogwheels, with 12 teeth (2), stiffly attached on the same axis, roll on the both gear wheel (3) on each site of the reduction. This avoids big torsion strains on the final piece of the reduction (4). A potentiometer is connected to the superior axis (7), and attached to the connectors (5) and to the final piece of the reduction (4).

The backlash of the reduction gearbox is about one degree and its output varies between $65 \%$ and $75 \%$ for an output torque ranging between 0.2 and $0.5[\mathrm{Nm}]$. The backlash, although quite significant, is acceptable for our application. Indeed, these modules are not designed for performing high precision task but rather for producing a high torque, so as to move forward the robot. The amplitude and the ordering of the joint rotations are much more crucial for enabling the robot locomotion than high precision (since the practical does not entail navigation).
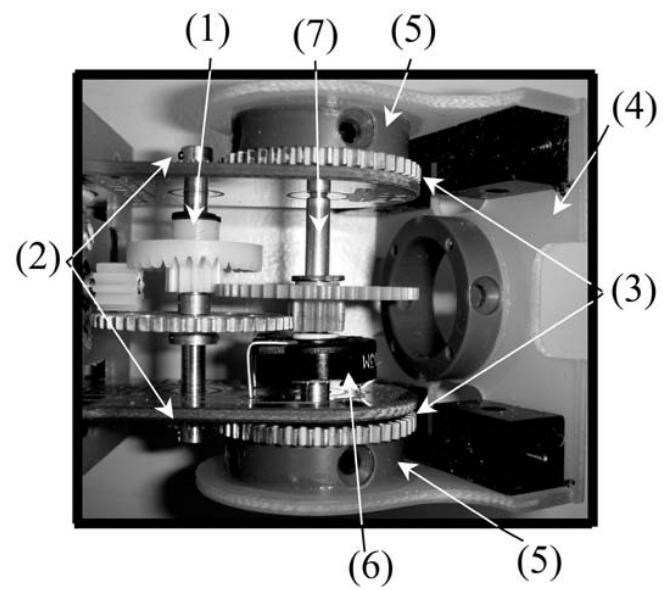

Figure 5: reduction gearbox.

\section{B. Electronics}

\section{1) Measures}

\section{a) Position}

The position of the rotating top can be measured in two ways, either using the potentiometer at the end of the reduction or using the encoder on the motor axis.

When using the potentiometer, the position is obtained by measuring the voltage at each side of its resistance with the AD converter of one of the microcontrollers. Since the position of the potentiometer relatively to the reduction axis is not precise, an offset must be tuned and added by the software. The potentiometer gives the absolute position after the reduction and is thus especially useful for initialization and security. However, it is noisy and not perfectly linear.

An encoder with 16 increments is mounted on the motor axis. Using a codage in quadrature yields 64 pulses for a complete revolution. since the ratio of the reduction gear is 192 , the resolution of the encoder is

$$
\frac{2 \cdot \pi}{192 \cdot 64}=511.32 \cdot 10^{-6}[\mathrm{rad}]
$$

This amounts to less than $1 / 30$ degree. As the backlash of the reduction gear is about one degree, this resolution is largely sufficient.

The position after the reduction gear can be approximated by the position of the motor axis divided by the reduction ratio. This yields only an approximate measure due to the backlash in the reduction gear. Moreover, the origin of the encoder must be initialised using the potentiometer, as the encoder only yields relative values.

The system composed of the amplifier and the motor is linear on a wide range of values. In comparison, the system composed of the amplifier, the motor, and the reduction gearbox is less linear, due to the hysteresis of the backlash in the reduction gearbox. Linearity is important for an efficient control if linear control theory methods are used as in this practical. Thus, using the reduction gear box in the control will result in a less stable control and can cause oscillations in the system.

\section{b) Current}

The current running through the motor is obtained by measuring the voltage on a shunt resistance.

\section{2) On-board microcontrollers}

There are two micro-controllers on the board:

- DsPic 30f4011

- Pic $16 f 876$

Both microcontrollers can be used to control the motor and for communication. One can use either of them or both in parallel. This can be useful if one wants to dedicate a controller to a specific task such as motor control, interfacing, trajectory generation, adaptation of control coefficients.

a) Firmware of Pic16f879 (The ASL I2C motor control module [1])

This firmware has been developed by [1] and allows to easily and quickly control a motor for a given application. It implements a PI torque or velocity control, as well as a PD position control with various options such trajectory generation (with squared, triangle or sinus profiles), output saturation, Anti-Reset Windup (ARW), and input inversion. It uses a I2C-based communication protocol with a bandwidth of about $100[\mathrm{~kb} / \mathrm{s}]$. 


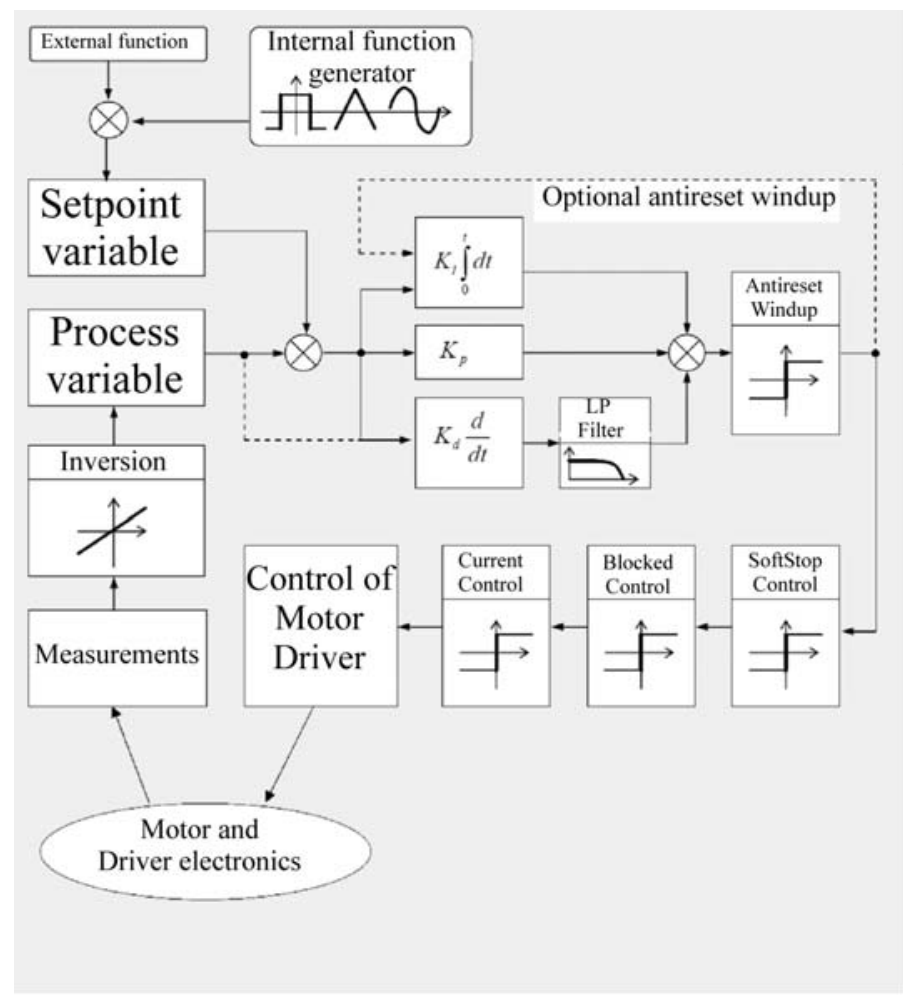

Figure 6: Dof-Box motor module functionality schema [1]. Adapted from [1].

\section{b) Firmware du DsPic30f4011}

The DsPic30f411 is an extension of the Pic16f879 described above. It has several additional peripherals such as UART and CAN modules for communication, QEI (Quadrature Encoder Interface) for position measurement through the encoder, AD conversion for position measurement through the potentiometer and a PWM module to fix the motor voltage through the amplifier. All those peripherals reduce the computational load of the processor.

Moreover, the DsPic firmware allows a PID control in position, velocity and torque and other linear or non-linear control. The structure of the code is similar to the one of the Pic (see Figure 6).

\section{Communication}

In the current application, the trajectory generation, the user interface and any other application runs on a master PC. This PC communicates with the robot through a RS232 cable connected to a I2C bus which runs through all the modules. Each module has a different address, so the master can send orders and data to specific modules through that address. The modules do not communicate directly with each other.

As mentioned above, we intend to add a CAN port managed by the DsPic. This will add in flexibility in the communication scheme, thanks to interesting properties of the CAN protocol such as multi-master communication, the priority system, high bandwidth and reliability. As the DsPic supports CAN and UART in parallel any module can be used as a UART/CAN converter.

Using the CAN communication protocol, one will add new possibilities such as direct inter-module communication. This way, particular modules could take control of the robot and send high priority messages such as security alerts. This is especially interesting in a complex robotic system.

\section{PRACTICALS}

The practicals last 12 hours split into three sessions of four hours. During the first session, the students are asked to characterize a Dof-Box and design the position PD control loop. During the last two sessions, the students are asked to design, assemble and program a robot with locomotion abilities. They should then test and analyze the abilities of their robot to react to various constraints

During the practicals, the students are provided with the following:

- 5 to 7 Dof-Box modules

- A computer

- 10 male type mechanical connectors

- A power supply

- A serial /I2C converter

- Measuring devices

- A screw-driver

1) First session

During the first session, the students go through all the steps necessary for the design of the control loop. Those steps are divided into four exercises:

a) Measuring the efficiency of the reduction gear box

The first exercise consists in measuring the friction torques in the reduction gear.

This is done by horizontally fixing a bar after the reduction gear. A small weight can be attached to this bar at various locations, thus creating a static torque of 0.1 to $0.5[\mathrm{Nm}]$. The motor is then rotated at constant speed. The current running through the motor is measured when the bar is horizontal and the generated torque can be computed. Looking at the plot of the results, the students should extract the static and the dynamic friction torques, see Fig 7. The latter is proportional to the mass and is used to compute the output of the reduction gear. 
Motor torque, as a function of reduction output torque (dynamic mode)

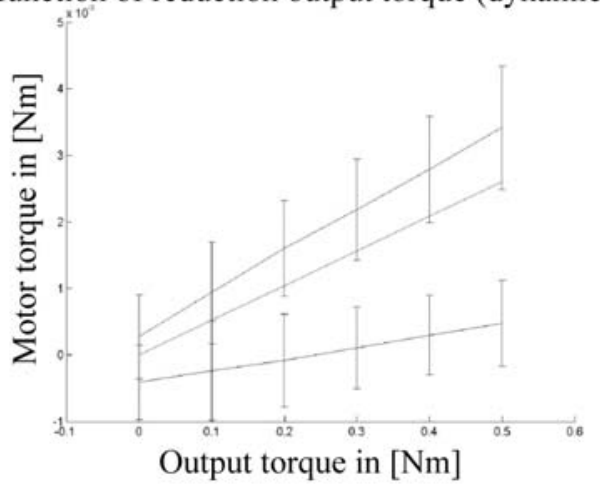

Figure 7: Torque generated by the motor as function of the weight after the reduction. The data is shown when moving upward (upper curve) and downward (lower curve). The middle curve is the theoretical curve when neglecting friction in the reduction gearbox. Measuring the backlash of the reduction gear.

b) Measuring the backlash of the reduction gear.

The aim of the second exercise is to compute the backlash in the reduction gear. Using the encoder, the students put the Dof Box in a particular configuration. For each such configuration (covering the whole range of motion), the students measure the backlash of a $50 \mathrm{~cm}$ long stick fixed after the reduction gear while manually immobilizing the first gear wheel. They can thus estimate the angular backlash for each angular position. With this exercise, the students become aware of the significant backlash in the reduction gear and of the resulting non-linearity of the potentiometer. They should thus conclude that it is better to use the encoder, which is not affected by this backlash.

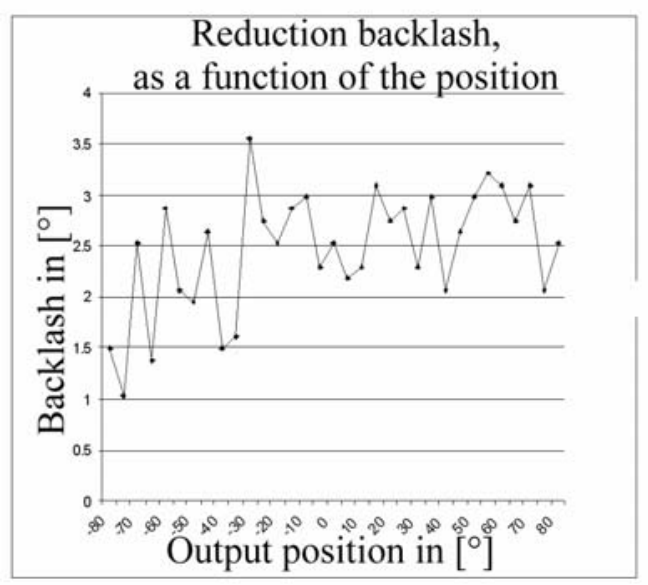

Figure 8: Backlash in the gearbox as a function of the postion ${ }^{1}$. c) Computing the transfer function of system

The third exercise aims at computing the transfer function of the system. First the students compute the equations of motion of the system, using the characteristics of the motor, the inertia of the weight after the reduction gear, and the reduction gear efficiency.

\section{d) Desing of the PD control loop}

In the fourth exercise, the students are asked to design a PD control loop, using the transfer function computed during the preceding exercise. The bandwidth of the closed-loop system must be about $15[\mathrm{~Hz}]$. The students have more information than required for this task, and so they need to figure out what exactly their needs are. An algebraic regulation method is suggested, but they can choose any method they want.

\section{2) Second and third sessions}

The first session allowed the students to determine the control parameters for an efficient control in most realizations of robots. However, thanks to high friction coefficients, the system is very stable, so even if the preceding exercise is not complete the control coefficient can be adapted "by hand" to have a working (although less efficient) robot.

During later two sessions, the students are asked to design, assemble and program their moving robot, using the control loop designed during the first session. First, they need to think of a shape that will enable their robot to move on an horizontal plane. They then need to assemble the robot and design joint angle trajectories required for moving. Using the $\mathrm{PC}$, they need to write a C-program that configures the modules (joint angle range and control parameters) through the serial cable. The program also computes joint trajectories and dynamically sends desired motor positions to the robot over the serial connection. Due to the large number of possible combinations, the 15 robots that were so far created in the framework of this practical were all different. Some examples of robots are shown in Figures 9 and 10. Figure 11 illustrates corresponding locomotion patterns.

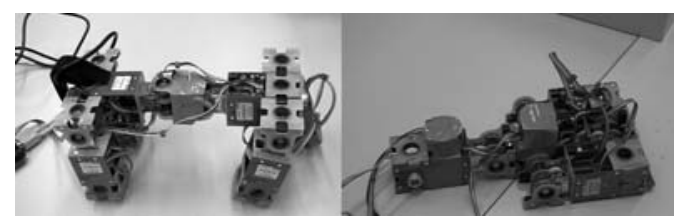

Figure 9: Dog and frog robots

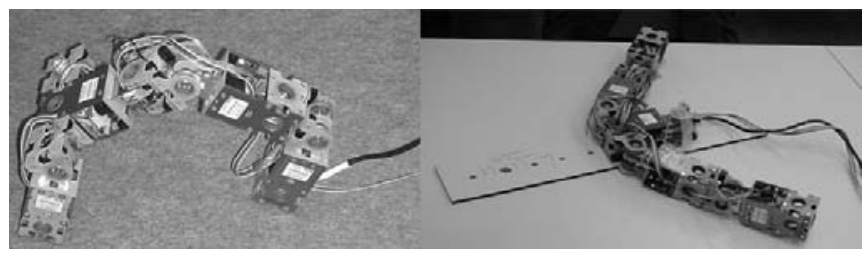

Figure 10: Tripod, rower robots

1 One can notice that after the first exercise, the backlash has increased from one two three degrees. This is due to a error in the design of a component, which increases its backlash when subjected to high torques. 


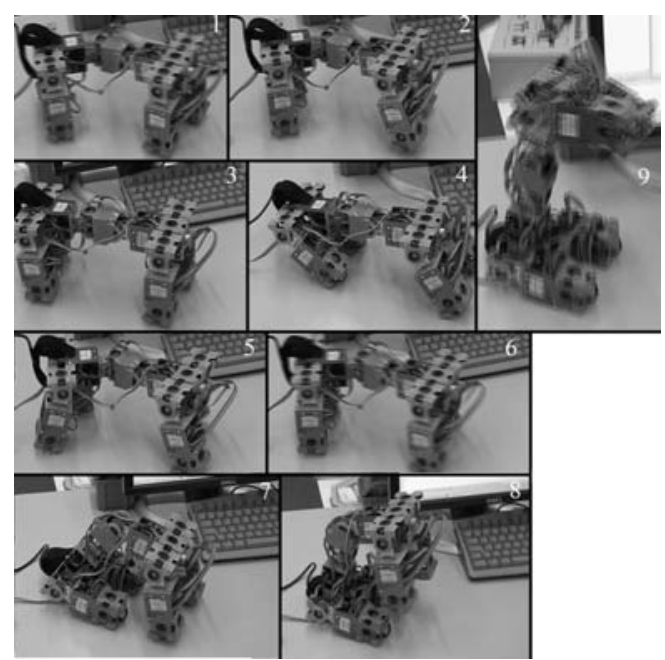

Figure 11: The dog robot can walk and sit

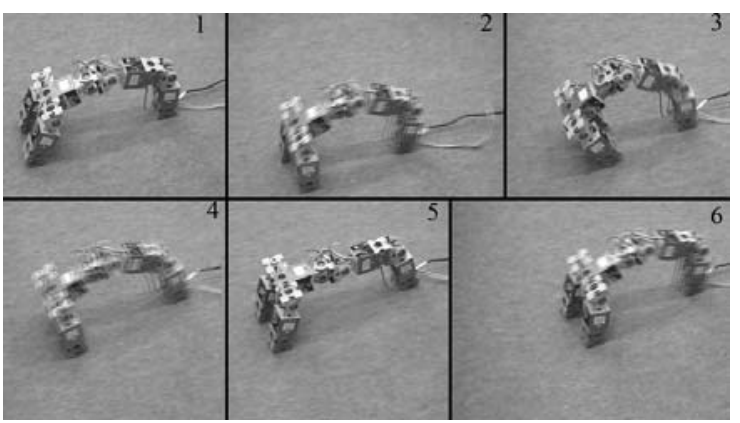

Figure 12: Locomotion pattern of the tripod robot

\section{CONCLUSION}

The Dof Box II modular robot has been developed as an educational tool for robotics practices. It has several interesting characteristics.

First, the number of possible mechanical connections makes this robot highly modular. Moreover, it is able to generate important torques. A dynamical torque of $1[\mathrm{Nm}]$ is enough to lift three serially connected modules, which allows the robots to have relatively complex limbs. Yet, this robot is easy to use, build and program. It takes only a few hours to build a moving robot that can be rather complex. It can be easily interfaced with a computer, through a basic serial cable.

The robot is also very robust. During the practicals, robots have stood important instantaneous torques (up to $2.5[\mathrm{Nm}]$ ), static torques (up to $1.5[\mathrm{Nm}]$ ) and dynamic torques (up to 0.7 $[\mathrm{Nm}]$ ) without damage. Finally, the robot is rather cheap, each module costs less than 120 \$. This makes it affordable for universities to purchase several of those as class equipment.

Some further improvements on the DOF BOX II are currently under consideration. These include automatic mechanical connections that would enable the robot to assemble and disassemble itself autonomously, on-board batteries, wireless communication, reducing the reduction gear backlash, miniaturization by the use of brushless motors, and reducing the inertia/power ratio to enable a module to lift more than three other modules.

This robot described in this paper is a very interesting tool for teaching the basics of robotics to students. Its modularity enables the students to easily build their own robot and control them. They develop their creativity and get confronted to typical engineering problems to which they need to bring their own solutions. This practical has become quite popular among students.

\section{ACKNOWLEDGMENT}

We thank all the persons of the ACORT and ACI workshops at EPFL for building the electronics boards and the people from the AEM and ATSE workshops at EPFL for the production of the mechanical components. We thank Micha Hersch for the English Translation of this paper.

\section{REFERENCES}

[1] D. Bär, "The ASL I2C motor control module" initial released revision, J. EPFL Lausanne: STI/ASL, 2003.

[2] F. Michaud, J-F. Laplante, H. Larouche, A. Duquette, S. Caron, D. Létourneau, P. Masson, “Autonomous Spherical Mobile Robot for Child Development Studies". IEEE Transactions on Systems, Man, and Cybernetics, 35(4):1-10.

[3] C. Eldershaw, M. Yim, D. Duff, K. Roufas, Y. Zhang, "Modular Self Reconfigurable Robots", Robotics for future land warfare seminar and workshop, Defence Science Technology Organisation, Adelaide, Australia, 2002.

[4] A. Kamimura, H. Kurokawa, E. Yoshida, S. Murata, K. Tomita and S. Kokaji, "Automatic Locomotion Design and Experiments for a Modular Robotic System”, IEEE/ASME Transactions on Mechatronics, Vol. 10, Issue 3, pp. 314-325, 2005.

[5] A. Castano, A. Behar, P. Will. "The Conro Modules for Reconfigurable Robots". IEEE/ASME Trans. Mechatronics, 7(4):403-409, December 2002.

[6] F. Mondada, B. Michael, “e-puck EPFL education robot”, http://www.epuck.org/, EPFL, Ecublens janvier 2007.

[7] A. Billard, B. Robins, K. Dautenhahn, and J. Nadel, "Building Robota, a Mini-Humanoid Robot for the Rehabilitation of Children with Autism". the RESNA Assistive Technology Journal. In Press, 2006 